\section{International Scientific Journal Theoretical \& Applied Science}

p-ISSN: 2308-4944 (print) e-ISSN: 2409-0085 (online)

Year: 2015 Issue: 03 Volume: 23

Published: 30.03.2015 http://T-Science.org
Gulmira Saginganovna Kenzhebekova $\mathrm{PhD}$ of the department of Higher Mathematics L.N. Gumilyov Eurasian National University, Kazakhstan gulmira0703@yandex.ru

SECTION 1. Theoretical research in mathematics.

\title{
ON THE INTEGRABILITY OF THE FUNCTIONS WITH MONOTONE DECREASING FOURIER COEFFICIENTS WITH RESPECT TO MULTIPLICATIVE SYSTEMS
}

Abstract: In this work one generalization of the theorem of Hardy-Littlewood about Fourier series with monotone coefficients with respect to multiplicative systems is proved.

Key words: Fourier series, Fourier coefficients, Dirichlet's kernel, multiplicative systems.

Language: Russian

Citation: Kenzhebekova GS (2015) ON THE INTEGRABILITY OF THE FUNCTIONS WITH MONOTONE DECREASING FOURIER COEFFICIENTS WITH RESPECT TO MULTIPLICATIVE SYSTEMS. ISJ Theoretical \& Applied Science 03 (23): 55-58.

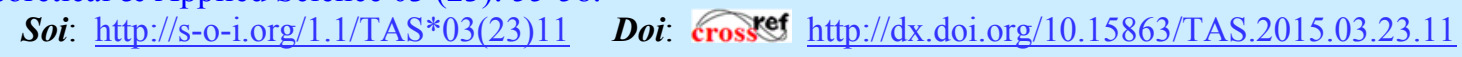

\section{ОБ ИНТЕГРИРУЕМОСТИ ФУНКЦИЙ С МОНОТОННО УБЫВАЮЩИМИ КОЭФФИЦИЕНТАМИ ФУРЬЕ ПО МУЛЬТИПЛИКАТИВНЫМ СИСТЕМАМ}

Аннотация: В данной работе доказывается одно обобщение теоремы Харди-Литтлвуда о рядах с монотонными коэффициентами Фурье по мультипликативным системам.

Ключевые слова: Ряды Фурье, коэффициенты Фурье, ядро Дирихле, мультипликативные системы.

Приведем определение мультипликативной системы Прайса.

Пусть $\left\{p_{n}\right\}_{n=1}^{\infty}-\quad$ последовательность натуральных чисел, $p_{n} \geq 2, n=1,2, \ldots$. Положим

$$
m_{0}=1, m_{n}=p_{1} \ldots p_{n} \text { при } n \in N .
$$

Тогда каждое $x \in[0,1)$ имеет разложение

$$
x=\sum_{n=1}^{\infty} \frac{x_{n}}{m_{n}}, 0 \leq x_{n}<p_{n}, \quad x_{n} \text { - целые. }
$$

Такое разложение будет единственным, если для $x=\frac{l}{m_{n}}$ брать разложение с конечным числом ненулевых $x_{n}$. Если $k \in Z_{+}$записано в виде $k=\sum_{s=1}^{v(k)} k_{s} m_{s-1}, 0 \leq k_{s}<p_{s}, k_{s}$ - целые, то по определению для $x \in[0,1)$ полагаем

$$
\chi_{k}(x)=\exp \left(2 \pi i \sum_{j=1}^{v(k)} \frac{x_{j} k_{j}}{p_{j}}\right)
$$

Таким образом, построенная система $\left\{\chi_{k}(x)\right\}_{k=1}^{\infty}$ называется мультипликативной на $[0,1)$ системой Прайса (см.[1]). Известно, что указанная система является полной ортонормированной системой в $L_{2}[0,1)$ (см.[2]).

Мультипликативные системы рассматривались впервые в работах Н.Я. Виленкина [3], А.В. Ефимова [4], А. Прайса [5]. Свойства мультипликативных систем излагаются в [2]. 
Сумма

$$
D_{n}(x)=\sum_{k=0}^{n-1} \chi_{k}(x)
$$

называется $n$-м ядром Дирихле. Известно, что

$$
D_{m_{n}}(x)=m_{n} \chi_{\left[0, \frac{1}{m_{n}}\right)},
$$

где $\chi_{E}$ - характеристическая функция множества $E$.

Г.Г. Харди и Дж. И. Литтлвуд доказали [6], что при убывании к нулю последовательности $\left\{b_{k}\right\}$ сумма ряда

$$
\sum_{k=1}^{\infty} b_{k} \cos k x, \quad \sum_{k=1}^{\infty} b_{k} \sin k x
$$

принадлежит $L_{p}[0,2 \pi], 1<p<\infty, \quad$ тогда и только тогда, когда

$$
\sum_{n=1}^{\infty} b_{n}^{p} n^{p-2}<\infty .
$$

Аналог этой теоремы для системы Уолша доказан Ф. Морицем [7], для мультипликативных систем с ограниченными образующими последовательностями $\left\{p_{n}\right\} \quad\left(1 \leq \sup _{n} p_{n}<M\right)$ М.Ф. Тиманом, К. Тухлиевым [8].

Многие авторы вводили более слабые условия на $b_{k}$, чем монотонность. А.А. Конюшков [9] изучал поведение наилучших приближений в $L_{p}[0,2 \pi], 1<p<\infty, \quad$ для функций, задаваемых тригонометрическими рядами (1), при условии, что $b_{k} k^{-\tau}$ убывает при некотором $\tau \geq 0$ и $\lim _{k \rightarrow \infty} b_{k}=0$. В другой форме это условие рассматривал О. Сас [10]. Г.К. Лебедь [11] изучал ряды (1) также при условии, что $k^{\tau} b_{k}$ возрастает при некотором $\tau>0$ и $\lim _{k \rightarrow \infty} b_{k}=0$.

Основной целью данной работы является доказательство следующей теоремы.

Теорема. Пусть функция $f \in L_{p}(1 \leq p<\infty)$ c монотонно убывающими коэффициентами Фурье $\quad b=b_{n}(f) \downarrow 0$ по мультипликативной системе $\left\{\chi_{k}(x)\right\}_{k=0}^{\infty} \quad$ и $\varphi(t)-$ неубывающая функция на $[0, \infty)$ такая, что $\varphi\left(t^{2}\right) \leq C \varphi(t)$ и $\varphi(t) t^{-\varepsilon}(\varepsilon>0)$ не возрастает.

Тогда для того, чтобы сходился интеграл

$$
\int_{0}^{1}|f(x)|^{r} \varphi\left(|f(x)|^{r}\right) d x
$$

необходимо и достаточно, чтобы сходился ряд

$$
\sum_{n=1}^{\infty} m_{n}^{r-1} b_{m_{n}}^{r} \varphi\left(m_{n-1}\right)
$$

Для доказательства теоремы нам понадобится следующая вспомогательная лемма (см.[12]).

Лемма. Пусть функция $\Phi(u)$ неотрицательна и не убывает на $[0 ; \infty)$. Тогда:

А) если неотрицательная функция $\psi(u)$ не возрастает на $(0,1]$ и

$$
A \equiv \int_{0}^{1} \psi(u) \Phi\left(\frac{1}{u}\right) d u<\infty,
$$

TO

$$
B \equiv \int_{0}^{1} \psi(u) \Phi(\psi(u)) d u<\infty ;
$$

В) если $\Phi\left(u^{2}\right) \leq C \Phi(u)$ при некоторой постоянной $C \geq 1$ и $u \in[0, \infty)$, то для всякой неотрицательной функции $\psi(x)$, измеримой на $[0,1)$ и удовлетворяющей условию (4), выполнено неравенство (5).

Доказательство теоремы.

Необходимость. В силу свойства ядра Дирихле $\quad D_{m_{n}}(t)$ для монотонных последовательностей $b_{n}(t)$ имеем

$$
\begin{gathered}
F\left(\frac{1}{m_{n}}\right)=\int_{0}^{\frac{1}{m_{n}}} f(t) d t=\frac{1}{m_{n}} \int_{0}^{1} f(t) D_{m_{n}}(t)= \\
=\frac{1}{m_{n}} \sum_{k=0}^{m_{n}-1} b_{k}(t) \geq b_{m_{n}} .
\end{gathered}
$$

Значит,

$$
b_{m_{n}} \leq \int_{0}^{\frac{1}{m_{n}}} f(t) d t, \quad n=1,2, \ldots
$$

Поэтому, учитывая то, что $\varphi$ неубывающая функция по условию, имеем 


$$
\begin{gathered}
\sum_{n=1}^{\infty} m_{n}^{r-1} b_{m_{n}}^{r} \varphi\left(m_{n-1}\right) \leq \\
\leq \sum_{n=1}^{\infty} m_{n}^{r-1} \varphi\left(m_{n-1}\right)\left(\int_{0}^{\frac{1}{m_{n}}} f(t) d t\right)^{r} \leq \\
\leq \sum_{n=1}^{\infty} m_{n-1}^{r}\left(\frac{2}{m_{n-1}}\right)\left(\int_{0}^{\frac{1}{m_{n-1}}} \varphi^{\frac{1}{r}}\left(\frac{1}{t}\right)|f(t)| d t\right)^{r} \leq \\
\leq \sum_{n=1}^{\infty}\left(\frac{1}{m_{n-1}}-\frac{1}{m_{n}}\right)\left(\frac{1}{m_{n-1}} \int_{0}^{\frac{1}{m_{n-1}}} \varphi^{\frac{1}{r}}\left(\frac{1}{t}\right)|f(t)| d t\right)^{r} \leq \\
\leq C_{r} \sum_{n=1}^{\infty} \frac{1}{m_{n-1}}\left\{\frac{1}{m_{n}}\left\{\frac{1}{x} \int_{0}^{x} \varphi^{\frac{1}{r}}\left(\frac{1}{t}\right)|f(t)| d t\right\}^{r} d x=\right. \\
=C_{r} \int_{0}^{1}\left\{\frac{1}{x} \int_{0}^{x} \varphi^{\frac{1}{r}}\left(\frac{1}{t}\right)|f(t)| d t\right\}^{r} d x .
\end{gathered}
$$

В силу известного неравенства Харди [13] имеем

$$
\sum_{n=1}^{\infty} m_{n}^{r-1} b_{m_{n}}^{r} \varphi\left(m_{n-1}\right) \leq \int_{0}^{1} \varphi\left(\frac{1}{t}\right)|f(t)|^{r} d t .
$$

Тогда согласно лемме из (2) вытекает (3). Достаточность. Для всякого $\varepsilon \in(0,1)$ имеем

$$
\begin{gathered}
\sum_{n=1}^{\infty} m_{n}^{r-1} b_{m_{n}}^{r} \varphi\left(m_{n}\right)= \\
\sum_{n=1}^{\infty} m_{n}^{-(1-\varepsilon)}\left\{b_{m_{n}} m_{n} \varphi^{\frac{1}{r}}\left(m_{n}\right) m_{n}^{-\frac{\varepsilon}{r}}\right\}^{r},
\end{gathered}
$$

то, используя известное неравенство [13]

$$
\begin{aligned}
& \sum_{n=1}^{\infty} m_{n}^{-c}\left(\sum_{v=1}^{n} a_{v}\right)^{l} \leq C(c, l) \sum_{n=1}^{\infty} m_{n}^{-c}\left(m_{n} a_{n}\right)^{l} \\
& \quad\left(c>1, l>1, a_{v} \geq 0\right) .
\end{aligned}
$$

и свойство функции $\varphi(t)$, получим

$$
\begin{gathered}
\sum_{n=1}^{\infty} b_{m_{n}}^{r} m_{n}^{r-1} \varphi\left(m_{n}\right) \geq \\
\geq C_{r} \sum_{n=1}^{\infty} m_{n}^{-(1-\varepsilon)}\left\{\sum_{v=1}^{n} b_{v} \varphi^{\frac{1}{r}}(v) v^{-\frac{\varepsilon}{r}}\right\} \geq \\
\geq C_{r} \sum_{n=1}^{\infty} \varphi\left(m_{n}\right) m_{n}^{-(1-\varepsilon)}\left(\sum_{v=1}^{m_{n}} b_{v}\right)^{r}= \\
=\sum_{n=1}^{\infty} \varphi\left(m_{n}\right) m_{n}^{-1}\left(\sum_{v=1}^{m_{n}} b_{v}\right)^{r}=S(r, \varphi) .
\end{gathered}
$$

Так как

$$
\begin{gathered}
J(r, \varphi)=\int_{0}^{1}|f(x)|^{r} \varphi\left\{\left.f(x)\right|^{r}\right\} d x= \\
=\sum_{n=1}^{\infty} \frac{1}{m_{n+1}} \int_{m_{n}}^{\frac{1}{m_{n}}}|f(x)|^{r} \varphi\left\{|f(x)|^{r}\right\} d x
\end{gathered}
$$

и для $\frac{1}{m_{n+1}} \leq x \leq \frac{1}{m_{n}}$

$$
\begin{gathered}
|f(x)| \leq \sum_{v=1}^{m_{n}} b_{v}+\frac{b_{n}}{x} \leq \\
\leq \sum_{v=1}^{m_{n}} b_{v}+\left(m_{n+1}\right) b_{n} \leq 3 \sum_{v=1}^{m_{n}} b_{v},
\end{gathered}
$$

то

$$
\begin{gathered}
J(r, \varphi) \leq 3^{r} \sum_{n=1}^{\infty} \int_{\frac{1}{m_{n+1}}}^{\frac{1}{m_{n}}}\left(\sum_{v=1}^{m_{n}} b_{v}\right)^{r} \varphi\left\{3 \sum_{v=1}^{m_{n}} b_{v}\right\}^{r} \leq \\
\leq 3^{r} \sum_{n=1}^{\infty} m_{n}^{-1}\left(\sum_{v=1}^{m_{n}} b_{v}\right)^{r} \varphi\left\{3 \sum_{v=1}^{m_{n}} b_{v}\right\}^{r} \leq \\
\leq 3^{r} \sum_{n=1}^{\infty} m_{n}^{-1}\left(\sum_{v=1}^{m_{n}} b_{v}\right)^{r} \varphi\left(3^{r} b_{1}^{r} m_{n}^{r}\right) .
\end{gathered}
$$

Из условия (3) вытекает конечность величины $S(r, \varphi)$, а в силу свойств функции $\varphi(t)$ и конечность величины $J(r, \varphi)$, т.е. (2).

Аналогичная теорема для рядов по косинусам ранее доказана М.Ф. Тиманом [14]. 


\section{References:}

1. Agaev GN, Vilenkin NY, Jafarli GM, Rubinshtein AI (1981) Multiplikativnye sistemy funktsii i garmonicheskii analiz na nulmernykh gruppakh. ELM, Baku, 1981, 180.

2. Golubov BI, Efimov AV, Skvortsov VA (1987) Ryady i preobrazovaniya Uolsha: Teoriya i primeneniya. - M. Nauka, 1987, 344.

3. Vilenkin NY (1947) Ob odnom klasse polnykh ortonormalnykh system. Izv. AN SSSR, Seriya matematika, 1947, T.12, $363-400$.

4. Efimov AV (1966) O nekotorykh approksimativnykh svoistvakh periodicheskikh multiplikativnykh ortonormirovannnykh system. Mat. sbornik 1966, T. 69, № 3, 354 370.

5. Price AJ (1957) Certain group of ortonormal step functions. Canad. J.Math. 1957, V. 9, № 3, pp. $413-425$.

6. Zigmund A (1965) Trigonometricheskie ryady, Moscow: "Mir", 1965, T. 1,2.

7. Moricz F (1981) On Walsh series with coefficients tending monotonically to zero. Acta Math., Acad. Sci. Hung-1981-v.38, №1-4., pp.183-189.
8. Timan MF, Tukhliyev K (1983) Svoistva nekotorykh ortonormirovannykh sistem. Izv. vuzov, Matematika, 1983, №9, 65 - 73 .

9. Konyushkov AA (1958) Nailuchshee priblizhenie trigonometricheskimi polinomami i koefficienty Fur'e, Mat. sbornik, 44(1958), 5384.

10. Szasz O (1948) Quasi-monotone series, Amer. J. Math., 70(1948), 203-206.

11. Lebed GK (1967) O trigonometricheskikh ryadakh s koefficientami, udovletvoryayushimi nekotorym usloviyam. Mat. sbornik, 74(1967), 100-118.

12. Ulyanov PL (1968) Vlozhenie nekotorykh klassov funktsii. Izv. AN SSSR, seriya matematika, 1968, T.32, $649-686$.

13. Hardy GH, Littlewood JE, Polya G (1934) Inequalities, Cambridge University Press, New York, 1934.

14. Timan MF (1982) Nekotorye dopolneniya $\mathrm{k}$ teoremam vlozheniya Ulyanova. № 1750-82, Dep. v VINITI, 1982, 15. 